\title{
ARTICLE
}

\section{In vivo genome editing with a small Cas9 orthologue derived from Campylobacter jejuni}

Eunji Kim ${ }^{1,2, \star}$, Taeyoung Koo ${ }^{1,3, \star}$, Sung Wook Park ${ }^{4,5, \star}$, Daesik Kim ${ }^{1,6}$, Kyoungmi Kim¹, Hee-Yeon Cho ${ }^{1}$, Dong Woo Song 2 , Kyu Jun Lee ${ }^{2}$, Min Hee Jung ${ }^{2}$, Seokjoong Kim², Jin Hyoung Kim ${ }^{4,5}$, Jeong Hun Kim ${ }^{4,5,7}$ \& Jin-Soo Kim ${ }^{1,3,6}$

Several CRISPR-Cas9 orthologues have been used for genome editing. Here, we present the smallest Cas9 orthologue characterized to date, derived from Campylobacter jejuni (CjCas9), for efficient genome editing in vivo. After determining protospacer-adjacent motif (PAM) sequences and optimizing single-guide RNA (sgRNA) length, we package the CjCas9 gene, its sgRNA sequence, and a marker gene in an all-in-one adeno-associated virus (AAV) vector and produce the resulting virus at a high titer. CjCas9 is highly specific, cleaving only a limited number of sites in the human or mouse genome. CjCas9, delivered via AAV, induces targeted mutations at high frequencies in mouse muscle cells or retinal pigment epithelium (RPE) cells. Furthermore, CjCas9 targeted to the Vegfa or Hifla gene in RPE cells reduces the size of laser-induced choroidal neovascularization, suggesting that in vivo genome editing with CjCas9 is a new option for the treatment of age-related macular degeneration.

\footnotetext{
${ }^{1}$ Center for Genome Engineering, Institute for Basic Science (IBS), Seoul 08826, Republic of Korea. ${ }^{2}$ ToolGen, Byucksan Digital Valley 6-cha, 219 Gasan Digital 1-ro, Geumcheon-gu, Seoul 08501, Republic of Korea. ${ }^{3}$ Department of Functional Genomics, University of Science and Technology, Daejeon 34113, Republic of Korea. ${ }^{4}$ Department of Biomedical Sciences, Seoul National University College of Medicine, Seoul 03080, Republic of Korea. ${ }^{5}$ FARB Laboratory, Biomedical Research Institute, Seoul National University Hospital, Seoul 03082, Republic of Korea. ${ }^{6}$ Department of Chemistry, Seoul National University, Seoul 08826, Republic of Korea. ${ }^{7}$ Department of Ophthalmology, Seoul National University College of Medicine, Seoul 03080, Republic of Korea. ${ }^{\star}$ These authors contributed equally to this work. Correspondence and requests for materials should be addressed to Je.H.K. (email: steph25@snu.ac.kr) or to J.-S.K. (email: jskim01@snu.ac.kr).
} 
G enome editing has recently been democratized by the development of RNA-guided programmable nucleases repurposed from the type II clustered regularly interspaced short palindromic repeats (CRISPR)/CRISPRassociated (Cas) adaptive immune system against invading genetic elements in eubacteria and archaea ${ }^{1}$. Cas 9 , the single effector protein component in the system, is complexed with CRISPR RNA (crRNA) and trans-activating crRNA (tracrRNA) or with single-guide RNA (sgRNA) composed of essential portions of crRNA and tracrRNA to form a sequence-specific RNA-guided endonuclease (RGEN $)^{2}$. A new RGEN with desired target specificity is readily prepared by replacing crRNA or sgRNA, which hybridizes with a target DNA sequence. Cas9 RGENs cleave chromosomal DNA in a targeted manner, enabling genetic modifications or genome editing in cells and whole organisms ${ }^{3-8}$.

Cas9 derived from Streptococcus pyogenes (SpCas9), the first Cas9 orthologue to enable targeted mutagenesis in human cells $3,5,6,9$, is still the most widely used among several Cas9 proteins available for genome editing. Owing to it large size (1,368 amino acids, $4.10 \mathrm{kbp}$; Fig. 1a), however, the SpCas9 gene and its sgRNA sequence cannot be packaged together into certain viral vectors such as adeno-associated virus $(\mathrm{AAV})^{10}$ for efficient delivery into cells in vivo. Instead, the SpCas9 gene alone can be packaged into a single AAV vector in vivo ${ }^{11,12}$. Alternatively, SpCas9 can be split into two parts ${ }^{13,14}$, each can be packaged into two AAV vectors ${ }^{15,16}$. However, split SpCas9 is less active than the intact SpCas9 (refs 13,14). Furthermore, codelivery of two AAV vectors is less efficient than the delivery of a single AAV vector in vivo.

As an alternative to SpCas9, Staphylococcus aureus Cas9 (SaCas9) can be used for genome editing ${ }^{17}$. SaCas9 is smaller (1,053 amino acids, $3.16 \mathrm{kbp})$ than SpCas9 and can be packaged, together with its sgRNA, into an AAV vector. Still, the SaCas9 gene cannot be packaged with a florescent reporter gene or two sgRNAs, essential for targeted chromosomal deletions or other rearrangements, into a single AAV vector in vivo. Furthermore, protospacer-adjacent motif (PAM) sequences recognized by SaCas9, 5'-NNGRRT-3', occur less frequently than those recognized by SpCas9, $5^{\prime}$-NGG-3', limiting targetable sites. In this regard, smaller Cas9 orthologues with different PAM sequences are highly desired to expand in vivo genome editing. Here, we present Campylobacter jejuni-derived Cas9 (CjCas9) for efficient genome editing in vitro and in vivo. CjCas9 is composed of 984 amino-acid residues (2.95 kbp) (Supplementary Fig. 1), much smaller than SpCas9 or SaCas9 (Fig. 1a), but has never been shown to induce targeted mutagenesis in human or other eukaryotic cells or organisms. In this study, we characterized PAM sequences and optimized the sgRNA length to utilize $\mathrm{CjCas} 9$ for genome editing in mice.

\section{Results}

Determination of PAM sequences recognized by CjCas9. First, we determined the PAM sequences recognized by CjCas9 in vitro (Fig. 1b). A PCR amplicon library containing a CjCas9 target sequence followed by randomized 10-base pair (bp) sequences was cleaved by CjCas9 (and SpCas9 as a control) and a sgRNA specific to the target sequence. Cleaved duplexes were subjected to deep sequencing to identify PAM sequences recognized by CjCas9. This assay revealed that CjCas9 recognized $5^{\prime}$-NNNNACAC- $3^{\prime}$ or $5^{\prime}$-NNNNRY AC- $3^{\prime}$ (where $\mathrm{R}$ and $\mathrm{Y}$ stands for purines and pyrimidines, respectively) as PAMs in vitro. Using a different in vitro assay, Fonfara et al. ${ }^{18}$ reported that the optimal PAM for CjCas9 was $5^{\prime}$-NNNNACA- $3^{\prime}$.
To confirm the PAM specificity in human cells, we performed cell-based reporter assays by co-transfecting plasmids encoding CjCas9 and its sgRNA and reporter plasmids containing the target site with variable PAM sequences between RFP and GFP sequences ${ }^{19}$ (Fig. 1c). Because the RFP sequence is fused to the GFP sequence out of frame in these reporter plasmids, cells express RFP but not GFP (RFP + GFP - ) in the absence of CjCas9. When CjCas9 cleaves the target site and induces small insertions or deletions (indels), the GFP sequence can be fused to the RFP sequence in frame. These reporter assays showed that CjCas9 cleaved target sites containing $5^{\prime}$-NNNNRYAC- $3^{\prime}$ PAM sequences in HEK 293 cells. Importantly, the cytosine nucleotide at the $3^{\prime}$-end was essential.

Optimization of CjCas9 sgRNA length. We next optimized the sgRNA length for CjCas9-mediated genome editing in human and mouse cells (Fig. 2; Supplementary Fig. 2; Supplementary Table 1). We co-transfected CjCas9 and a series of sgRNAs with variable lengths into HEK 293 cells or NIH 3T3 cells and measured indel frequencies using targeted amplicon sequencing. The first nucleotide in the sgRNAs was fixed to an extra guanine (G) because sgRNAs are transcribed under the control of the U6 promoter, which requires a guanine at the $5^{\prime}$ end. Unlike SpCas9, which is most active with $\mathrm{GX}_{20}$ sgRNAs that hybridize with a 20-nucleotide target DNA sequence upstream of a PAM, CjCas9 was most active with $\mathrm{GX}_{22}$ sgRNAs that hybridize with a 22 nucleotides target sequence. $\mathrm{GX}_{20}$ and $\mathrm{GX}_{19}$ sgRNAs failed to induce indels at 8 out of 19 sites and 3 out of 4 sites, respectively in human or mouse cells.

We tested $\mathrm{GX}_{22}$ sgRNAs at 12 sites containing the optimal $5^{\prime}$-NNNNACAC- $3^{\prime}$ PAM in human cells. All of these sgRNAs co-transfected with CjCas9 were able to induce indels at frequencies that ranged from 1.0 to $64 \%(21 \pm 5 \%$, on average). CjCas9 also induced indels at sites with $5^{\prime}$-NNNNGCAC- $3^{\prime}$ PAMs, $5^{\prime}$-NNNNGTAC-3' PAMs, and 5'-NNNNATAC- $3^{\prime}$ PAMs albeit less efficiently $(10 \pm 3 \%, 10 \pm 4 \%$, and $16 \pm 5 \%$, respectively).

Genome-wide target specificities of CjCas9. We then determined genome-wide specificities of CjCas9 using nucleasedigested whole-genome sequencing (WGS; Digenome-seq) ${ }^{20-22}$ Cell-free genomic DNA digested with CjCas9 in vitro was subjected to WGS. Uniform cleavage patterns corresponding to on-target and off-target cleavage sites were computationally identified. CjCas9 designed to target 6 different DNA regions cleaved between one and 27 sites ( $7 \pm 4$ sites, on average) in the human or mouse genome (Fig. 3a-d). In parallel, we used Digenome-seq to test three SpCas9 nucleases designed to cleave sites that overlapped with CjCas9 target sites. SpCas9 cleaved 15 to 147 sites ( $70 \pm 40$ sites, on average) in the human or mouse genome, in line with our previous results showing that SpCas9 targeted to 11 different sites cleaved $90 \pm 30$ sites in the human genome $e^{21}$. One target site in the human genome contained a $5^{\prime}$-NGGNACAC-3' PAM recognized by both CjCas9 and SpCas9. CjCas9 and SpCas9 cleaved human genomic DNA at 5 sites and 45 sites, respectively, although the two Cas9 orthologues showed comparable indel frequencies at this particular site. Thus, the higher specificity of CjCas9 was not gained at the expense of its lower editing efficiency. Strikingly, two CjCas9 nucleases targeting the Rosa26 locus or the Vegfa gene in the mouse genome cleaved genomic DNA only at the single on-target site, reminiscent of the remarkable specificity of Cpf1 nucleases ${ }^{22,23}$. Sequence logos obtained computationally by comparing in vitro cleavage sites 
a
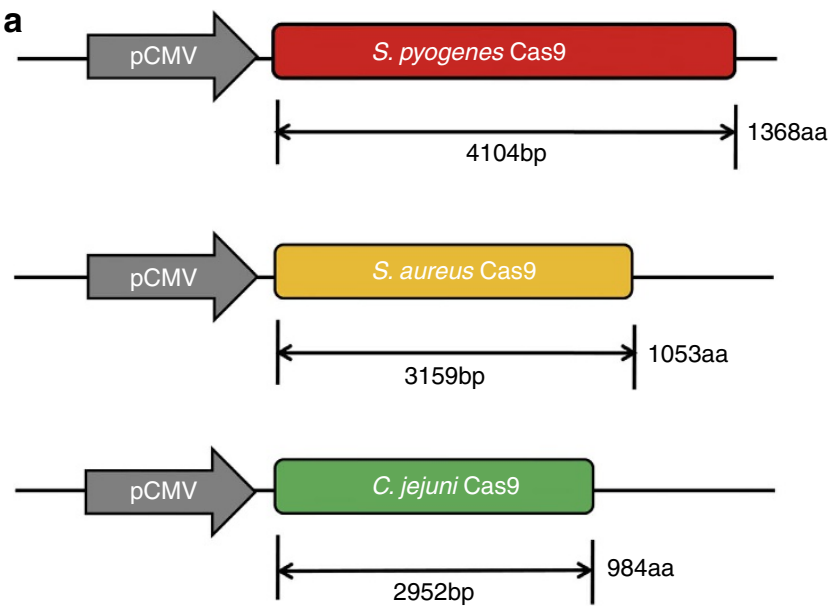

b
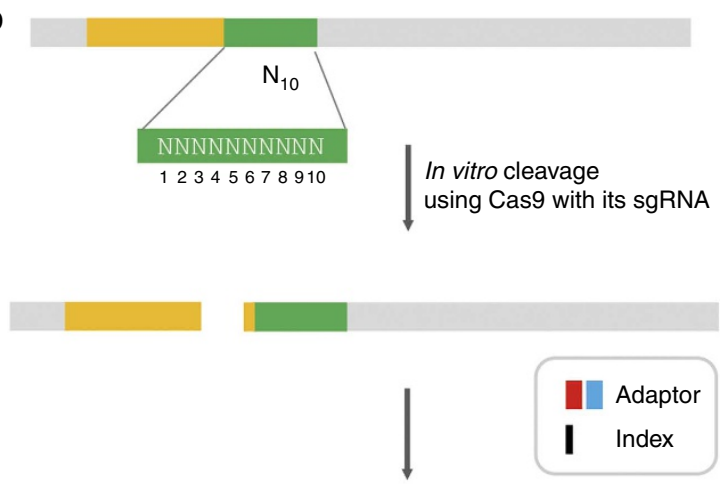

NGS library construction

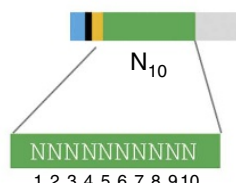

PAM identification using NGS data
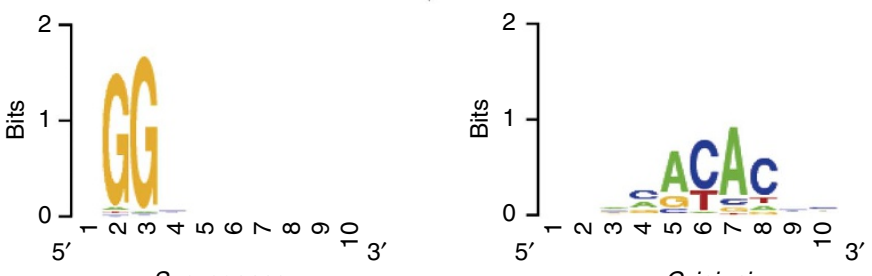
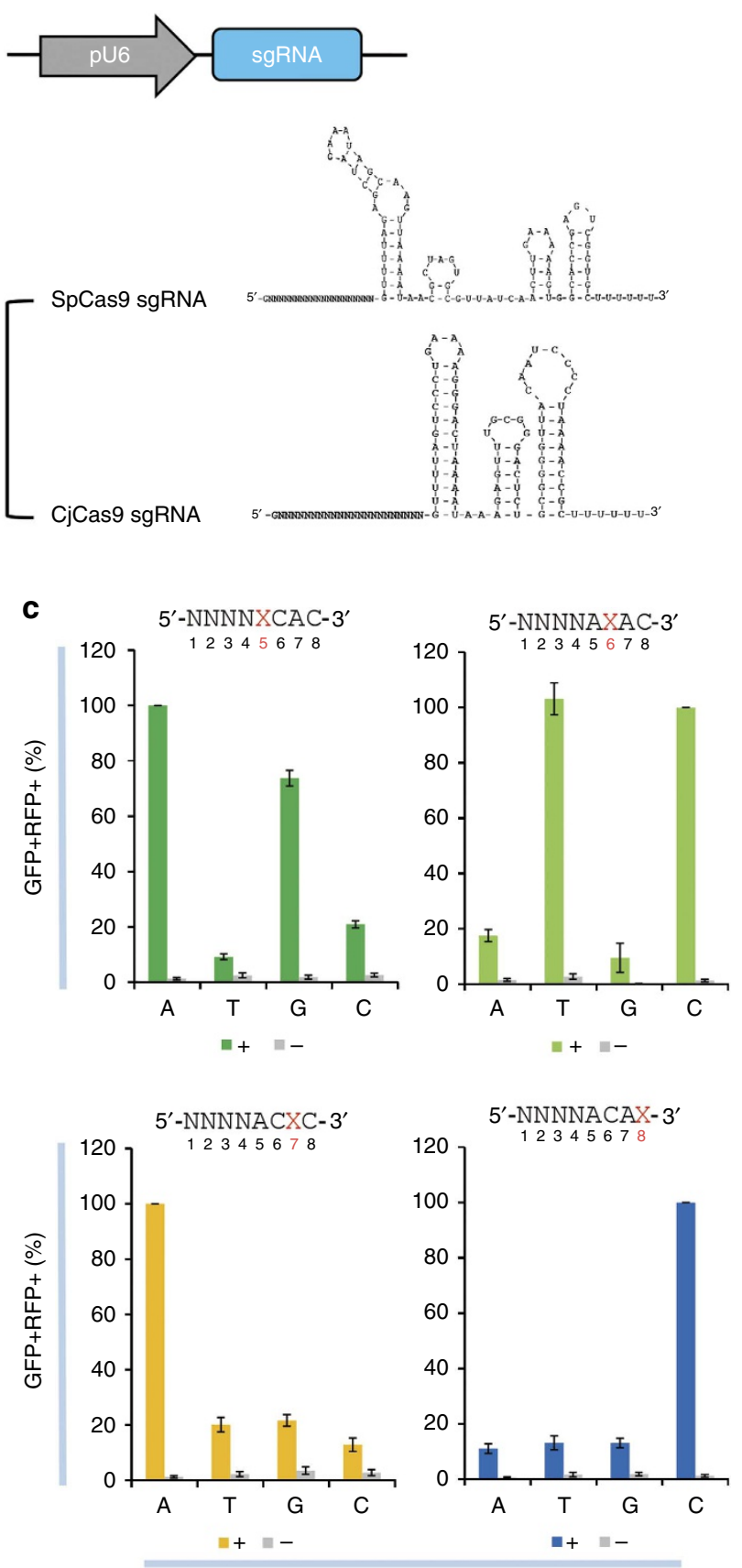

Nucleotide at position $\mathrm{X}$

Figure 1 | CjCas9 and its PAM specificity. (a) Cas9 orthologues and their sgRNAs. sgRNA structures were obtained using the mfold web server. See also Supplementary Fig. 1. (b) Schematic showing a PAM assay for characterization of PAM sequences recognized by Cas9. PCR amplicons containing a Cas9 target sequence (yellow) followed by randomized 10-bp $\left(\mathrm{N}_{10}\right)$ sequences (green) were cleaved by CjCas9 in vitro. The sequence logo showing the PAM specificity was obtained using deep-sequencing data. (c) PAM specificity in cells. Surrogate reporters encoding GFP and RFP and containing a CjCas9 target sequence with degenerate PAMs were transfected with CjCas9 and its sgRNA plasmid into HEK 293 cells. The percentage of cells that express both GFP and RFP was normalized with that obtained using the optimal reporter containing the 5'-NNNNACAC-3' PAM sequence. ( - ) represents a mock control. Error bars indicate s.e.m. $(n=3)$.

with each other unambiguously showed that these sites contained $5^{\prime}$-NNNNACAC- $3^{\prime}$ or $5^{\prime}$-NNNNRYAC-3' PAMs.

We chose the most promiscuous CjCas9 nuclease, which cleaved genomic DNA at 27 sites, and performed targeted amplicon sequencing to measure indel frequencies in human cells (Supplementary Table 2). Indels occurred at the on-target site but were not detectably induced at the other in vitro cleavage sites. Taken together, these results show that CjCas9 nucleases are highly specific in human cells. The remarkable specificity of CjCas9 can be at least partially attributed to its extended, 22 nucleotides target and 4 nucleotides PAM sequences, compared to the 20 nucleotides target and 2 nucleotides PAM sequences recognized by SpCas9.

Efficiency and specificity of CjCas9. We compared genome editing efficiencies and specificities of CjCas9 with those of SaCas9. We chose 3 overlapping sites in the human genome with $5^{\prime}$-NNGRRTAC-3' PAM sequences, which can be targeted by 


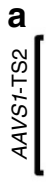

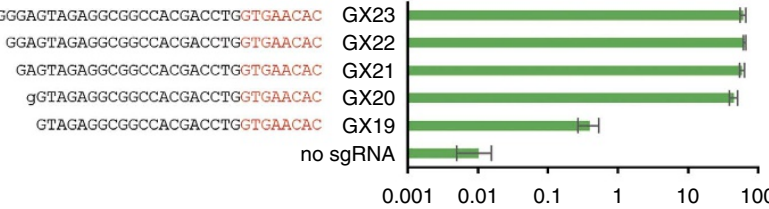

站
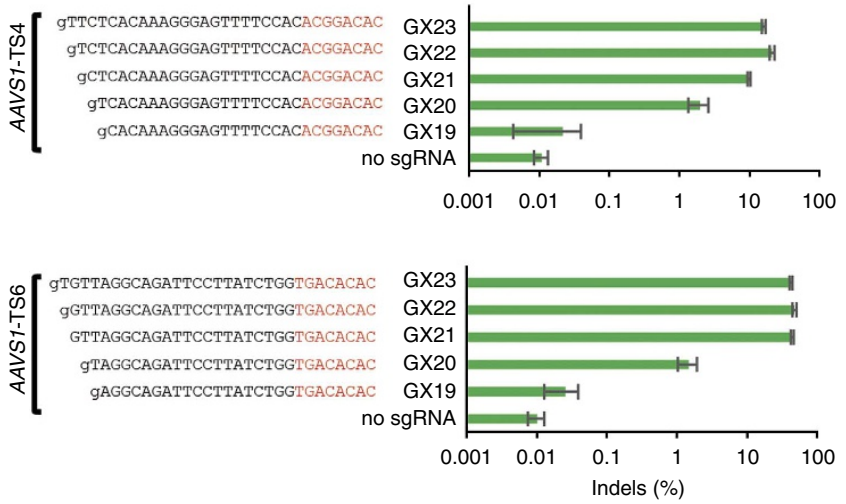

b

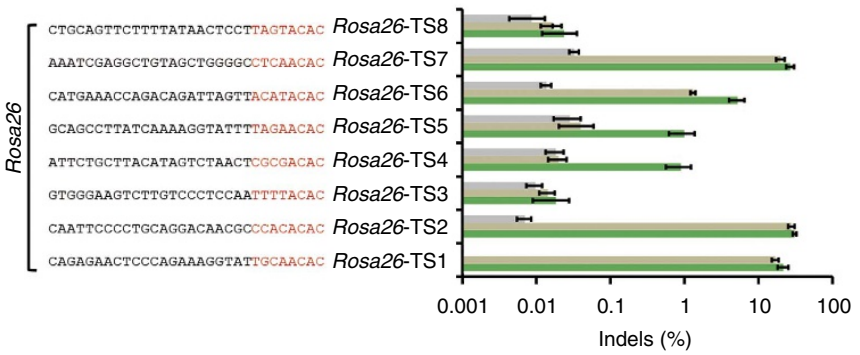

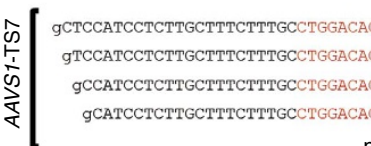

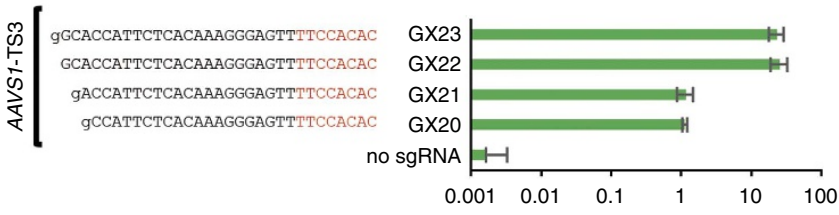

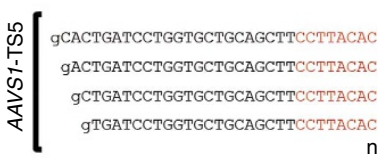
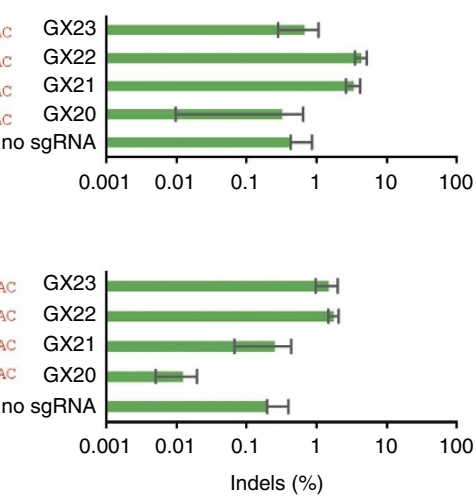

C
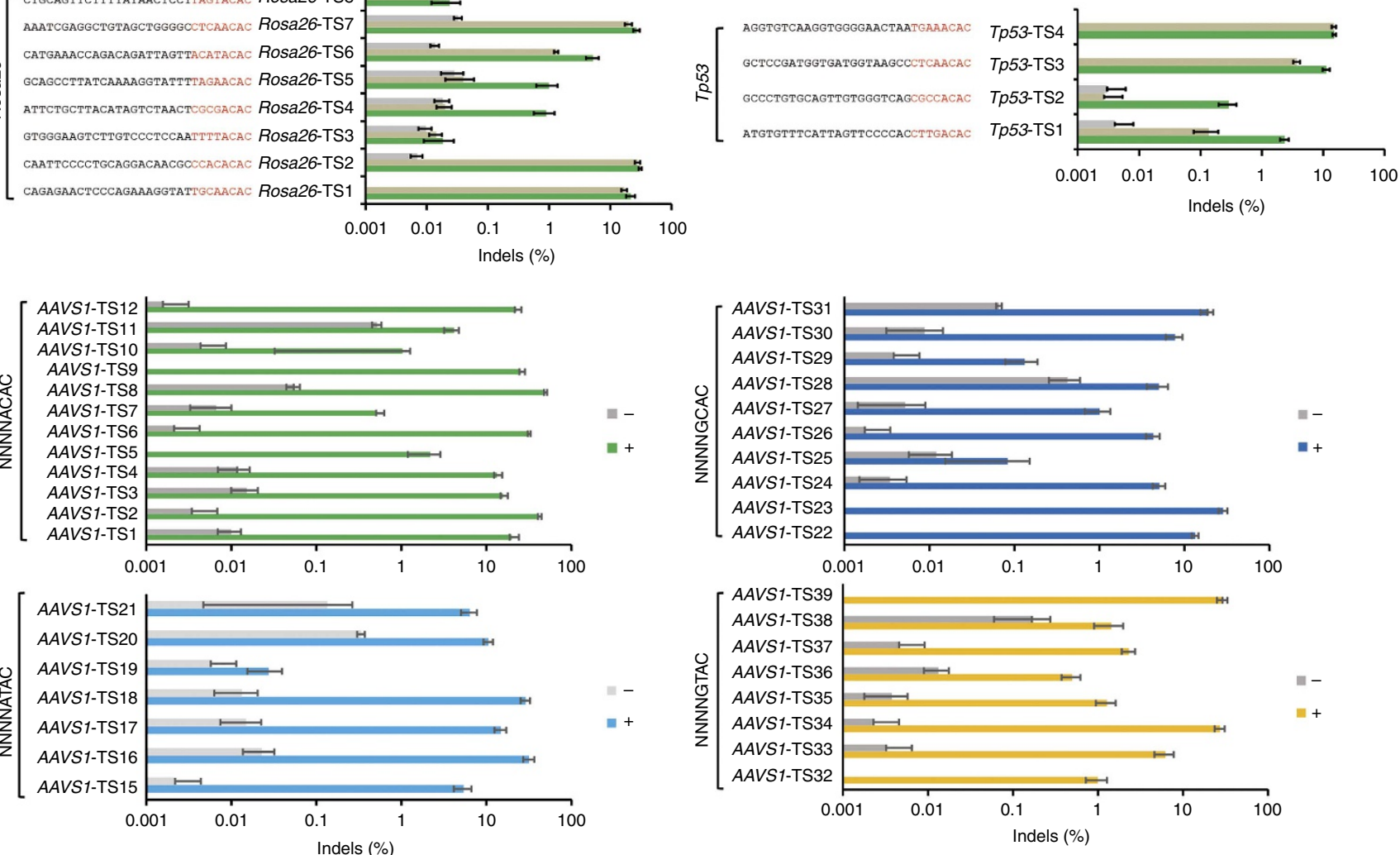

Figure 2 | Optimization of sgRNA length for CjCas9. (a) sgRNAs with variable lengths (19 to 23 nucleotide complementary with a target DNA sequence) were designed and transfected with CjCas9 plasmid into human HEK 293 cells. Genomic DNA was isolated $48 \mathrm{~h}$ after transfection. Indel frequencies were analysed by targeted deep sequencing. The first guanine nucleotide at the $5^{\prime}$ end that does not match the target sequence is shown in lower case. PAM motifs are shown in red. Error bars indicate s.e.m. $(n=3)$. (b) Mutation frequencies at target sites in the mouse genome. Rosa26 and Tp53-specific $\mathrm{gX}_{22}$ guide RNAs were designed and transfected into mouse NIH 3T3 cells together with CjCas9 plasmid. Genome editing efficiencies were examined by deep sequencing using genome DNA isolated from cells after $48 \mathrm{~h}$ of transfection. PAM motifs are shown in red. Error bars indicate s.e.m. ( $n=3$ ). See also Supplementary Fig. 2. (c) CjCas9-mediated genome editing at the human AAVS1 locus with different PAM sequences. sgRNAs targeting sites with a 5'-NNNNACAC-3' PAM (green; 12 sgRNAs), 5'-NNNNATAC-3' PAM (light blue; 7 sgRNAs), 5'-NNNNGCAC-3' PAM (dark blue; 10 sgRNAs), and $5^{\prime}$-NNNNGTAC-3' PAM (yellow; 8 sgRNAs) were designed and their activities examined in HEK293 cells with deep sequencing. Error bars indicate s.e.m. $(n=3)$. 
a

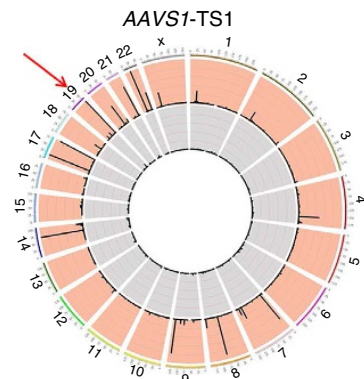

(-) Nuclease (+) CjCas9

b

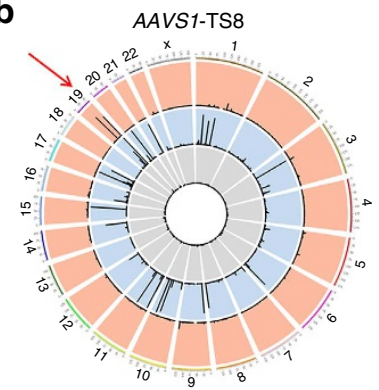

(-) Nuclease (+) CjCas9 (+) SpCas9

C

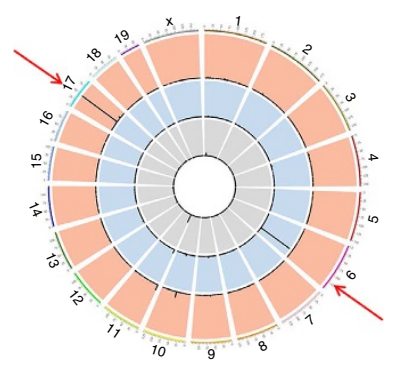

(-) Nuclease

(+) CjCas9_Rosa26-TS2

(+) CjCas9_Vegfa-TS13

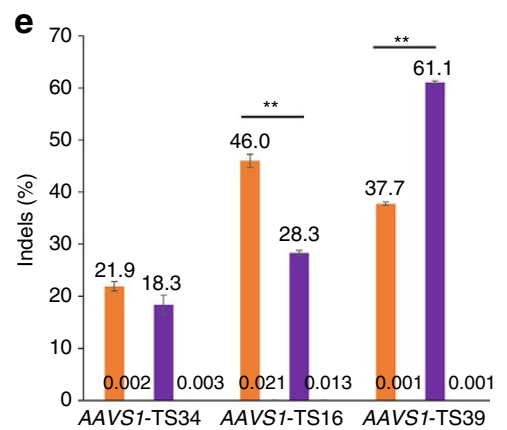

$=(+)$ CjCas9 $\quad$ ॥ (+) SaCas9

- (-) CjCas9

(-) SaCas9
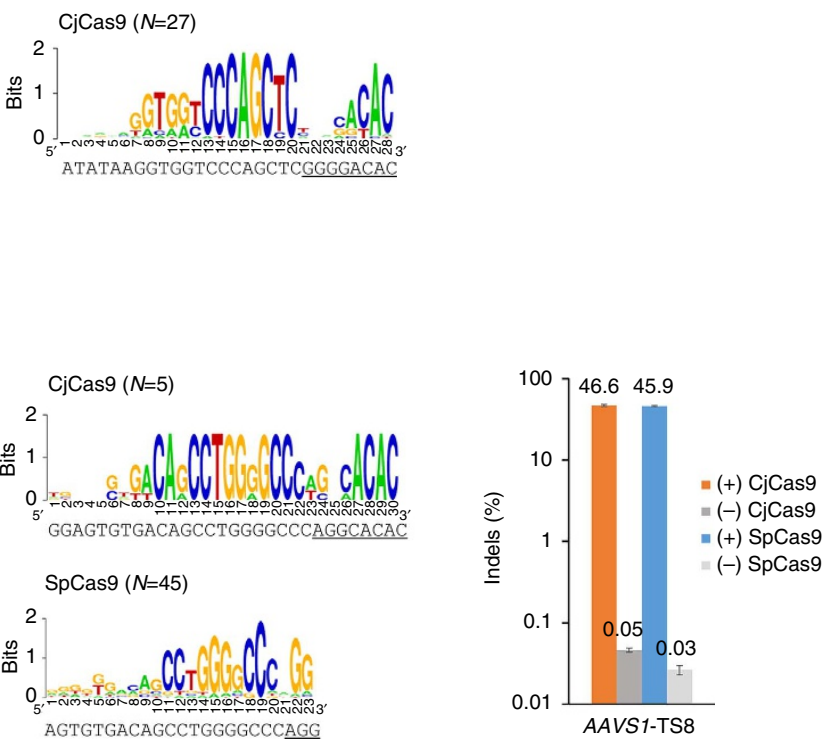

d
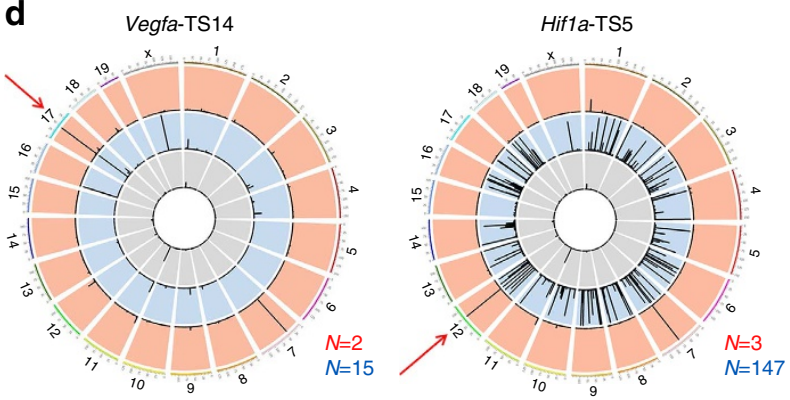

(-) Nuclease (+) CjCas9 (+) SpCas9
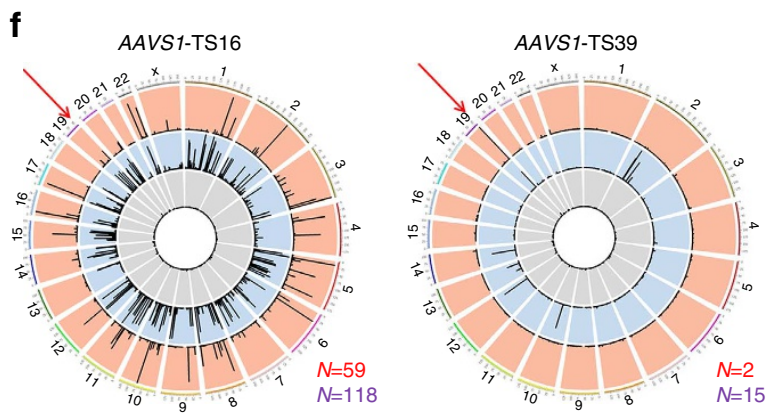

(-) Nuclease (+) CjCas9 (+) SaCas9

Figure 3 | Genome-wide target specificities of CjCas9 nucleases examined using Digenome-seq. Human or mouse genomic DNA isolated from HeLa cells or NIH 3 T3 cells (grey), respectively, was digested in vitro by Cas9 and its sgRNA targeted to the human AAVS1 locus (a,b,f) and the mouse Rosa26 (c), Vegfa (c,d) and Hif1a loci (d) and subjected to whole-genome sequencing. Circos plots show genome-wide DNA cleavage scores across the human or mouse genome. Red arrows indicate on-target sites. $N$ indicates the number of in vitro cleavage sites identified by Digenome-seq. (a,b) Sequence logos were obtained by comparing DNA sequences at in vitro cleavage sites with each other. (b) Indel frequencies at the AAVS1-TS8 site measured using targeted deep sequencing. CjCas9 (orange) or SpCas9 (blue) targeted to the AAVS1-TS8 site was transfected into human HEK293 cells. Error bars indicate s.e.m. $(n=3)$. (e) Indel frequencies at three AAVS1 sites targeted by CjCas9 (orange) and SaCas9 (violet).

both CjCas9 and SaCas9. CjCas9 and SaCas9 generated indels at these sites in HEK 293 cells with comparable frequencies of $35 \pm 7 \%$ and $36 \pm 13 \%$, respectively (Fig. $3 \mathrm{e}$ ). We then determined genome-wide specificities of CjCas9 and SaCas9 nucleases targeted to two of these overlapping sites using Digenome-seq (Fig. 3f). CjCas9 and SaCas9 targeted to the AAVS1-TS16 site cleaved human genomic DNA at 59 and 118 sites, respectively. CjCas9 and SaCas9 targeted to the AAVS1-TS39 site were more 
a

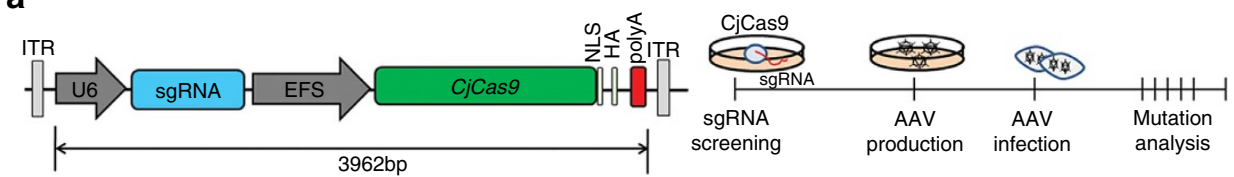

b

C
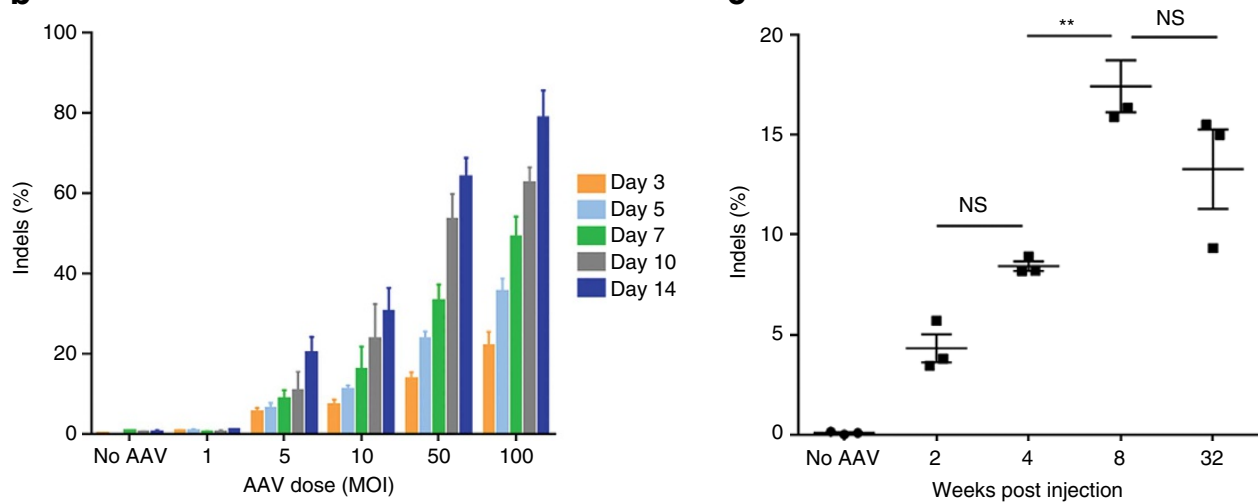

d

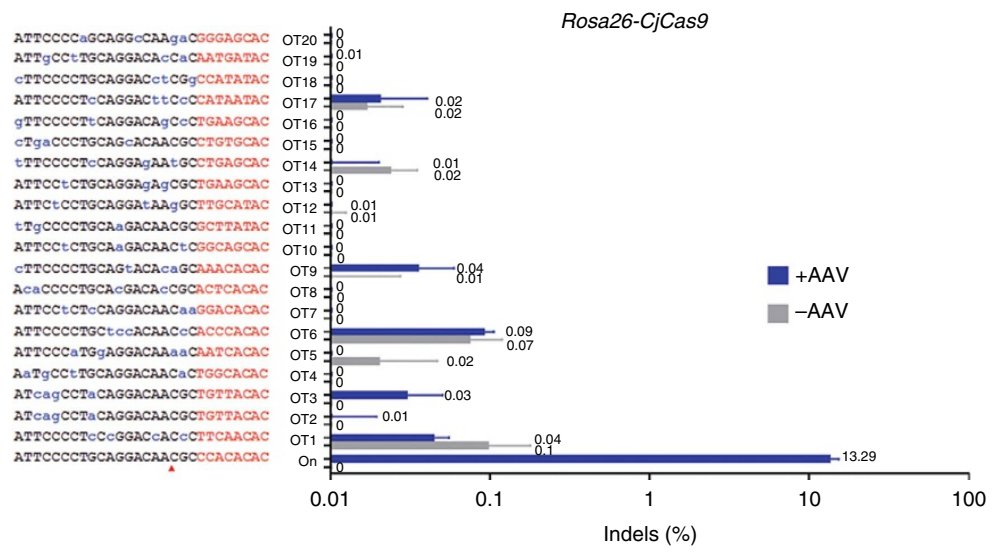

Figure 4 | AAV-mediated mutagenesis in vitro and in vivo. (a) AAV vector encoding CjCas9 and its sgRNA. (b) Indel frequencies at the Rosa26 target site in mouse $\mathrm{C} 2 \mathrm{C} 12$ myotubes infected with AAV-CjCas9. (c) Indel frequencies at the Rosa26 target site in TA muscles of C57BL6J mice injected with AAVCjCas9 measured at 2, 4, 8 and 32 weeks after injection. One-way ANOVA and Tukey's post hoc tests, ${ }^{\star \star} P<0.01$, NS, not significant. See also Supplementary Fig. 3. (d) No off-target indels were detected at 20 homologous sites that differed from the on-target site by up to 4 nucleotides in the mouse genome. Genomic DNA isolated from AAV-CjCas9-injected TA muscles of C57BL6J mice mice at 32 weeks after injection was analysed by targeted deep sequencing. Mismatched nucleotides are shown in blue and PAM sequences in red. Red arrows indicate cleavage positions within the 20-bp target sequences. Error bars indicate s.e.m. $(n=3)$.

specific than were those targeted to the TS16 site, cleaving human genomic DNA at 2 and 15 sites, respectively. Note that CjCas9 targeted to the TS16 site was more efficient than SaCas9 in terms of on-target indel frequency ( $46 \%$ versus $28 \%$ ), suggesting that the higher specificity of CjCas9 was not gained at the expense of a lower on-target efficiency. These results demonstrate that CjCas9 is as efficient as SaCas9 but is more specific than SaCas9 at least at the sites we tested in human cells.

All-in-one AAV vector for in vivo genome editing. With its small size, the CjCas9 gene plus a sgRNA sequence can be packaged into an all-in-one AAV vector (Fig. 4a). CjCas9 directed to the Rosa26 locus was expressed in $\mathrm{C} 2 \mathrm{C} 12$ mouse myotubes using an AAV serotype DJ (AAVDJ) vector. Indels accumulated at the target site in a time- and dose-dependent manner with a frequency of up to $79 \pm 7 \%$ (Fig. $4 \mathrm{~b}$ ). Because no sites other than the on-target site were cleaved by this particular CjCas9 nuclease in the mouse genome, as shown above using Digenome-seq, we identified, using Cas-OFFinder ${ }^{24}$, potential off-target sites that differed from the on-target site by up to 4 nucleotides in the genome. No indels were detected at the resulting 20 homologous sites by targeted deep sequencing even at day 14 post-infection (Supplementary Fig. 3; Supplementary Table 3), confirming the high specificity of this CjCas9 nuclease.

We next packaged the muscle-specific Spc512 promoter-driven CjCas 9 and the U6 promoter-driven Rosa26-specific sgRNA into a muscle-tropic AAV serotype 9 (AAV9) vector ${ }^{25}$ (Fig. 4c). The resulting virus was administered via intramuscular injection into tibialis anterior (TA) muscles of C57BL6J mice ( $n=3$ per group). CjCas9-induced indels were observed at the target site in TA muscles with a frequency of $17 \pm 1 \%$ and $13 \pm 2 \%, 8$ weeks and 32 weeks, respectively, after injection. No indels were detectably induced at the 20 possible off-target sites in TA muscles even 32 weeks after injection (Fig. $4 \mathrm{~d}$ and Supplementary Table 3), although CjCas9 was still expressed (Supplementary Fig. 4). This result suggests that a long-term expression of CjCas9 via $\mathrm{AAV}$ in vivo does not necessarily aggravate off-target effects. 
In vivo genome editing in the mouse retina. To show that CjCas9 can be expressed via $\mathrm{AAV}$ in other tissues such as retina in mice and to investigate the therapeutic potential of CjCas9-mediated gene surgery for the treatment of age-related macular degeneration (AMD), a leading cause of blindness in adults, we prepared an AAV9 vector encoding CjCas9 under the control of the elongation factor-1 short (EFS) promoter, enhanced green fluorescent protein (eGFP) linked to the $\mathrm{C}$ terminus of CjCas9 with the self-cleaving T2A peptide, and a U6 promoter-driven sgRNA specific to the Vegfa or Hifla gene, whose expression in the retina is associated with choroidal neovascularization $(\mathrm{CNV})^{26,27}$ (Fig. 5a,b). We monitored the expression of CjCas9 in the eye and measured indel frequencies using targeted deep sequencing and VEGFA protein levels using ELISA, 6 weeks after the resulting viruses were administered into the eye via intravitreal injection.

In retinal pigment epithelium (RPE) cells, primary target cells for the treatment of AMD, AAV encoding the Vegfa-specific CjCas9 (AAV-CjCas9: Vegfa) achieved indels with frequencies that ranged from 22 to $30 \%$ at day 14, 28, and 42 post injection (Fig. 5c). As expected, CjCas9-linked eGFP was expressed in RPE cells (Fig. 5d and Supplementary Fig. 5). At day 42 post injection, CjCas9-induced indels were observed at Rosa26, Vegfa, and Hifla target sites in the retina with a frequency of $44 \pm 18 \%$, $20 \pm 5,58 \pm 12$, respectively (Fig. 5e) and in RPE cells with a frequency of $14 \pm 5 \%, 22 \pm 3 \%$, and $31 \pm 2 \%$, respectively (Fig. 5f). As expected, the VEGFA protein level measured using ELISA was decreased in the retina treated with AAV encoding the Vegfa- or Hifla-specific CjCas9 (AAV-CjCas9: Vegfa and Hif1a) but not in those treated with AAV-CjCas9: Rosa26 (Fig. 5g). The VEGFA protein level was also decreased in RPE cells treated with the AAV-CjCas9: Vegfa. However, the protein level was not decreased in those with the AAV-CjCas9: Hifla or Rosa26 (Fig. 5h), suggesting that VEGFA expression is differentially regulated in the retina and in RPE cells. We did not measure HIF$1 \alpha$ protein levels because HIF- $1 \alpha$ is degraded under normoxia conditions. No off-target indels were detectably induced in these cells at one or two in vitro cleavage sites captured by Digenomeseq using the Vegfa-specific or the Hifla specific sgRNA, respectively (Fig. 5i), ruling out the possibility that the partial suppression of VEGFA expression in the retina and RPE cells were caused by CjCas9 off-target effects.

Therapeutic genome editing for the treatment of CNV. Next, we induced $\mathrm{CNV}$ in the eye by laser treatment 6 weeks after injection of AAV and measured the area of CNV 1 week later (Fig. 6a). Both AAV-CjCas9: Vegfa and AAV-CjCas9: Hifla reduced the area of CNV by $24 \pm 4 \%$ and $20 \pm 4 \%$, respectively, compared to the AAV-uninjected negative control (Fig. 6b-d). The Rosa26-specific CjCas9, used as another negative control, did not show any therapeutic effect. We noted that CjCas9 linked eGFP was expressed in RPE cells surrounding CNV, which are a major source of VEGFA in laser-induced CNV (Fig. 6b). This result shows that the reduction of $\mathrm{CNV}$ area coincides with targeted Vegfa mutagenesis and partial suppression of VEGFA expression in RPE cells (Fig. 5h).

We then investigated whether the partial gene knockout of $V$ egfa and Hifla in RPE cells using AAV caused any side effect. It was reported that a conditional knockout of the Vegfa gene but not that of the Hifla gene in mouse RPE cells leads to cone dysfunction ${ }^{28}$. We measured cone function using full-field electroretinography (ERG) in laser-untreated CNV-free mice 8 weeks after injection of AAV-CjCas9: Vegfa and AAV-CjCas9: Hifla (Fig. 6e,f). No significant decrease in photopic response (Fig. 6e) or $30 \mathrm{~Hz}$ flicker response (Fig. 6f) was observed in these mice, compared to AAV-uninjected control mice. We also measured the size of the opsin-positive area, which is closely related to cone function, in contact with RPE cells expressing CjCas9. The AAV-CjCas9: Vegfa reduced the size by $30 \pm 10 \%$, compared with the AAV-uninjected control (Fig. 6g,h; Supplementary Fig. 6), suggesting that the partial Vegfa gene knockout using AAV can still cause local opsin dysfunction near Vegfa-edited RPE cells. As expected, however, the AAV-CjCas9: Rosa26 or AAV-CjCas9: Hif1a did not cause any such cone dysfunction. Taken together, these results raise a concern about targeted inactivation of Vegfa in RPE cells using AAV and suggest that Hifla could be inactivated without causing cone dysfunction to avoid neovascularization for the treatment of AMD.

\section{Discussion}

In this report, we have presented a small and highly specific Cas 9 orthologue, derived from $C$. jejuni, that can be packaged with a reporter gene in an AAV vector for efficient gene surgery in vivo. The small size of CjCas9, compared with other orthologues including Neisseria meningitidis Cas9 (ref. 29), Streptococcus thermophilus Cas9 (ref. 29), SpCas9 (ref. 30) and SaCas9 (refs 17,31; Fig. 1a), allows more room for additional effectors or homology arms required for homologous recombination in AAV and other animal or plant viral vectors. Because CjCas9 is highly specific in vitro and in vivo, we expect that it will be widely used for precision genome editing in research and gene surgery in medicine.

In this study, we delivered the CjCas9 gene, its sgRNA sequence, and the GFP-coding gene to mutate three genes in two different tissues, TA muscles and eyes, in mice. CjCas9-induced indels at high frequencies in the Vegfa and Hifla genes in vivo. HIF- $1 \alpha$ is a hypoxia-inducible transcription factor that activates the transcription of VEGF A (ref. 32). Unlike VEGF A, a secretory protein and a primary therapeutic target for the treatment of AMD, HIF- $1 \alpha$ has not been considered as a drug target: Indeed, HIF1 $\alpha$ in particular and transcription factors in general cannot be targeted directly by antibodies or aptamers or small molecules. In this study, we showed that CjCas9 targeted to the Hifla gene in mouse eyes inactivated the gene in RPE cells efficiently and reduced the area of $\mathrm{CNV}$ in a mouse model of AMD. Because the CjCas9 target site in the mouse Hifla gene is perfectly conserved in the human HIF1A gene, the AAV presented in this study or its variants could be used for the treatment of human patients in the future. We expect that CjCas9 can be directed to other traditionally 'undruggable' genes or non-coding sequences to broaden the range of therapeutic targets, making the entire human genome potentially druggable.

\section{Methods}

Animals. The care, use, and treatment of all animals in this study were in strict agreement with the ARVO statement for the Use of Animals in Ophthalmic and Vision Research and College of Veterinary Medicine and the guidelines established by the Seoul National University Institutional Animal Care and Use Committee, which granted permission to perform animal experiments. Eight-week-old, male, specific pathogen-free C57BL/6J mice $(n=3-9)$ were used in this study. Mice were maintained under a $12 \mathrm{~h}$ dark-light cycle.

Laser-induced CNV model. After mice were anaesthetized, pupils were dilated with an eye drop containing phenylephrine $(0.5 \%)$ and tropicamide $(0.5 \%)$. Laser photocoagulation was performed using an indirect head set delivery system (Iridex) and laser system (Ilooda). Laser parameters were $810 \mathrm{~nm}$ wave length, $200 \mu \mathrm{m}$ spot size, $800 \mathrm{~mW}$ power and $70 \mathrm{~ms}$ exposure time. Laser burn was induced three to four times around the optic disc. Only burns that produced a bubble without vitreous haemorrhage were included in the study. Seven days later, the eyes were fixed in $4 \%$ paraformaldehyde for $1 \mathrm{~h}$ at room temperature. RPE complexes (RPE/choroid/ sclera) were prepared for immunostaining and then incubated with isolectin-B4 (Thermo Fisher Scientific, cat. no. I21413, 1:100) and anti-GFP antibody 
a

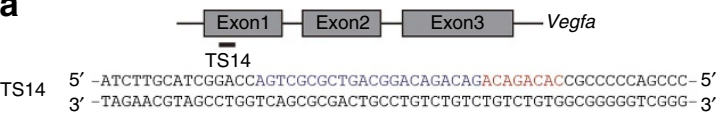

- Exon4 Exon5 - Exon6 - Hif1a

$\mathrm{T} \overline{\mathrm{S}} 5$

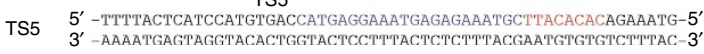

C

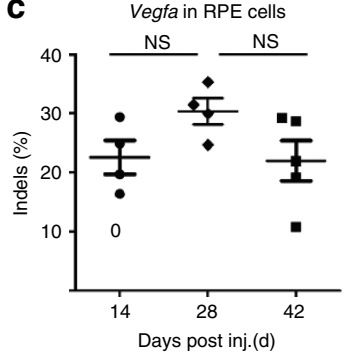

d

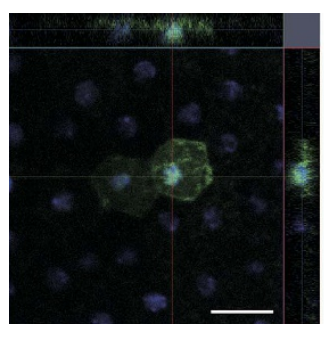

e

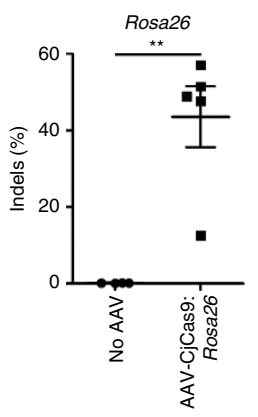

f

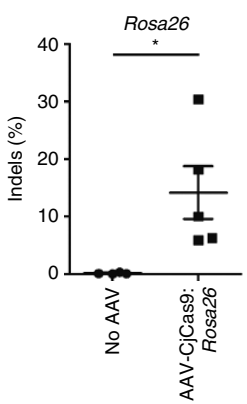

i

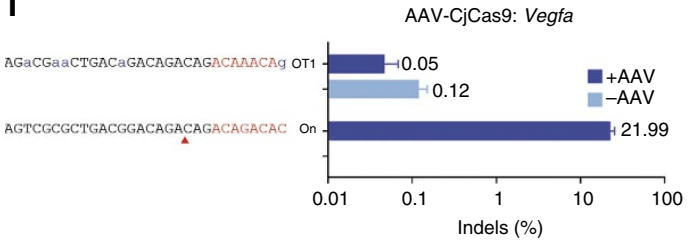

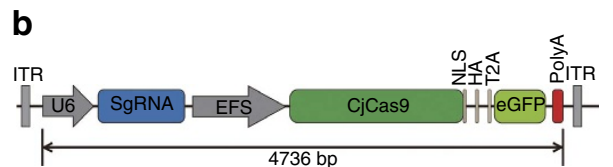
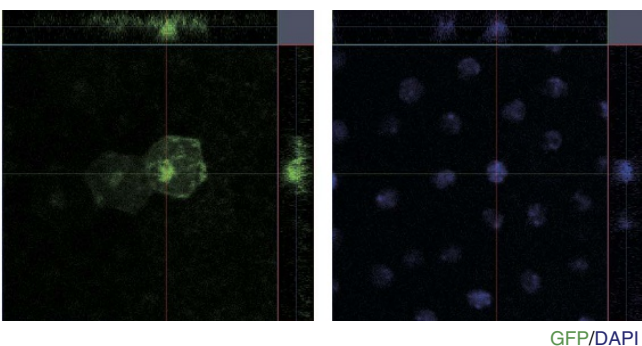

$\mathbf{g}$
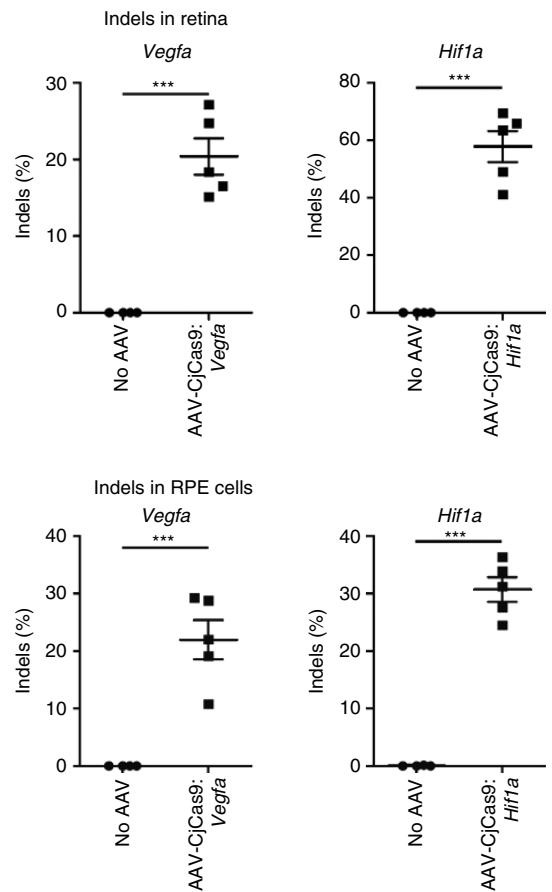

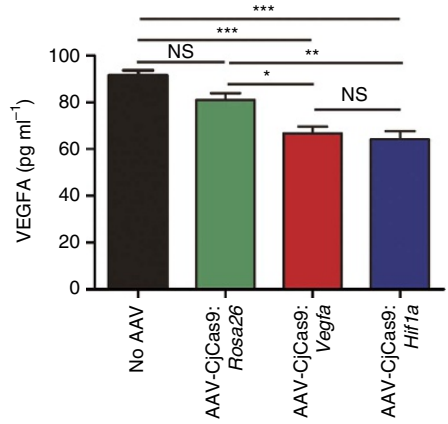

h

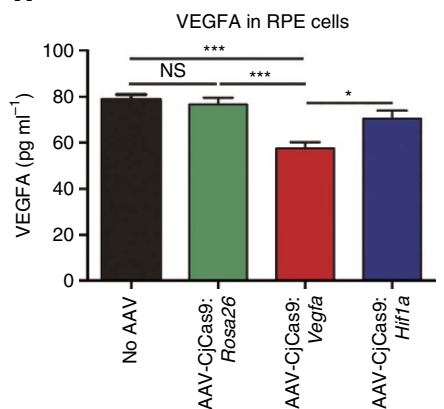

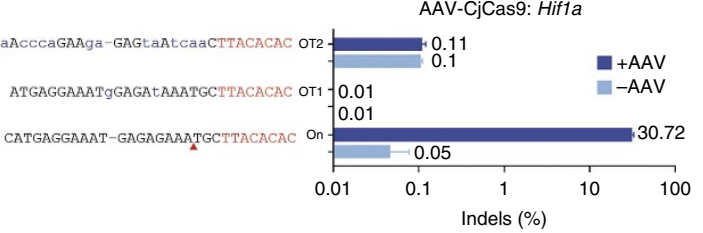

Figure 5 | In vivo genome editing with CjCas9 in the retina and retinal pigment epithelium. (a) The CjCas9 target sequences in Vegfa and Hifla/HIF1A genes. The PAM sequence and the sgRNA target sequence are shown in red and blue, respectively. (b) All-in-one AAV vector encoding CjCas9. (c) Indel frequencies at the Vegfa target site were analysed in RPE cells using deep sequencing at day 14, 28 and 42 post-intravitreal injection of AAV-CjCas9: Vegfa. Error bars indicate s.e.m. $(n=4-5)$. One-way ANOVA and Tukey's post hoc tests, NS, not significant. (d) Representative confocal images of in vivo eGFP expression in RPE cells of AAV-CjCas9-injected mice 6 weeks after injection $(n=6)$. eGFP was stained with anti-GFP antibody (green). Nuclei were counter-stained with DAPI (blue). Scale bar, $20 \mu \mathrm{m}$. (e-i) At day 42 post injection of AAV-CjCas9, indel frequencies and Vegfa protein levels were measured in retina and RPE cells using deep sequencing and ELISA, respectively. (e,f) Indel frequencies at the Rosa26, Vegfa and Hif1a target sites in the retina (e) and RPE cells (f). Error bars indicate s.e.m. ( $n=4$ for AAV-uninjected control, $n=5$ for AAV-CjCas 9$)$. Student's $t$-tests, ${ }^{\star} P<0.05$, ${ }^{\star \star \star} P<<0.001$. $(\mathbf{g}, \mathbf{h})$ VEGFA levels measured by ELISA in the retina $(\mathbf{g})$ and RPE cells (h), respectively. Error bars indicate s.e.m. $(n=6-7)$. One-way ANOVA and Tukey's post hoc tests, ${ }^{\star} P<0.05,{ }^{\star \star \star} P<0.001$. (i) Indel frequencies at in vitro cleavage sites identified by Digenome-seq. Genomic DNA isolated from RPE cells treated with AAV-CjCas 9 at 6 weeks post injection was subjected to targeted deep sequencing. Mismatched nucleotides are shown in blue and PAM sequences in red. Red arrows indicate cleavage positions within the 22-bp target sequences. 
(Abcam, ab6556, 1:100) overnight at $4{ }^{\circ} \mathrm{C}$. The RPE complex was flat-mounted and viewed with a fluorescent microscope (Eclipse $90 \mathrm{i}$, Nikon) or a confocal microscope (LSM 710, Carl Zeiss) at a magnification of $\times 100$. The CNV area was measured using Image J software (1.47v, NIH) by blinded observers. An average of 3-4 CNV areas per eye were analysed. Each group consisted of 17-18 eyes.
Construction of cjCas9 and sgRNA plasmids. A human codon-optimized CjCas9-coding sequence, derived from Campylobacter jejuni subsp. Jejuni NCTC 11168, was synthesized with a nuclear localization signal and an HA epitope at its C-terminal end (GeneArt Gene Synthesis, Thermo Fisher Scientific) and cloned into the p3s plasmid ${ }^{4}$. The trans-activating crRNA (tracrRNA) sequence and the

a

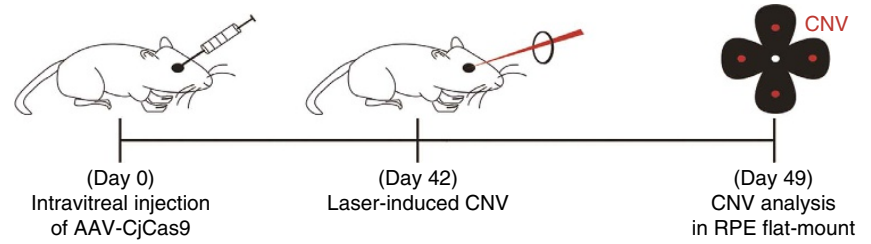

b

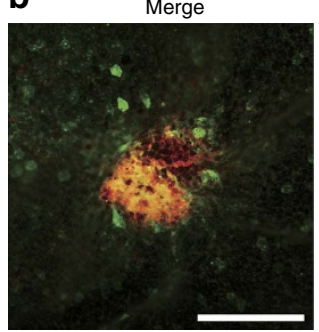

C

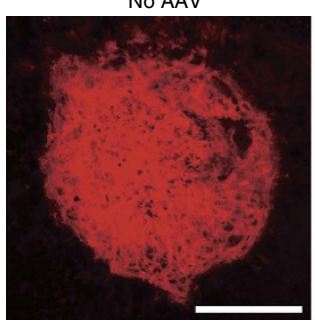

d

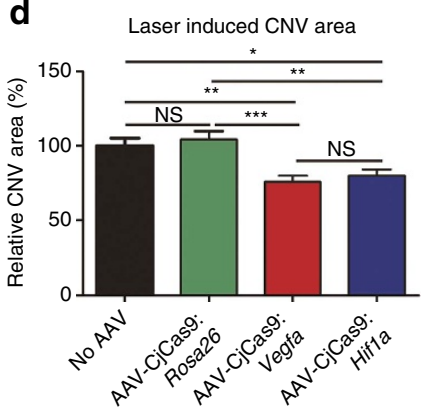

g

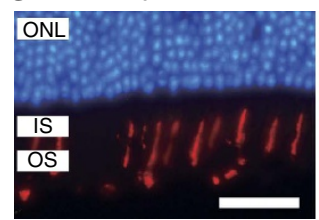

AAV-CjCas9: Rosa26

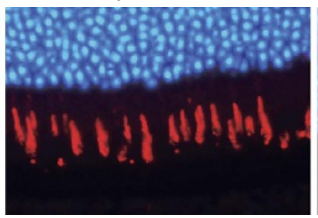

AAV-CjCas9: Vegfa

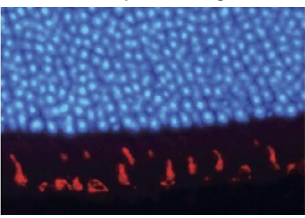

h

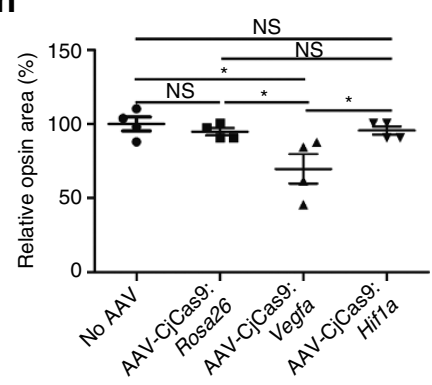

GFP

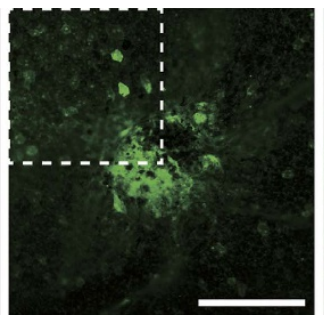

AAV-CjCas9: Vegfa

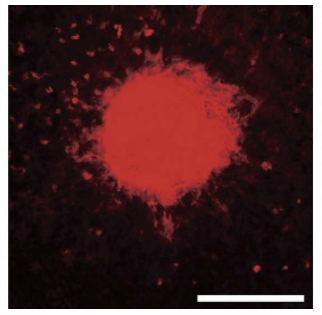

f
GFP-enlarged

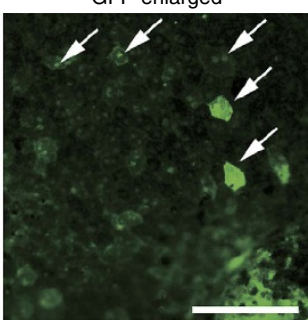

AAV-CjCas9: Hif1a

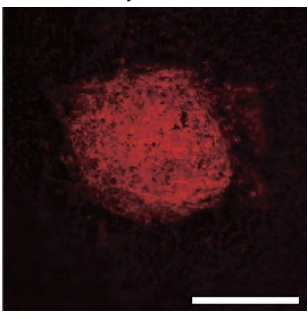

$30 \mathrm{~Hz}$ flicker response
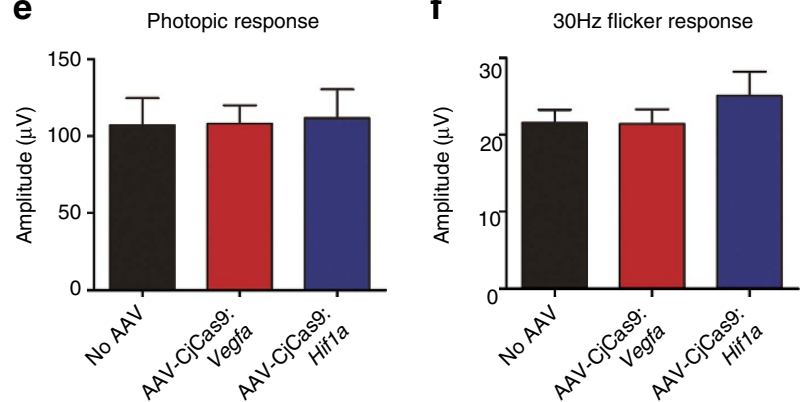

AAV-CjCas9: Hif1a

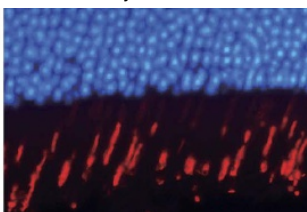

Opsin/DAPI 
precursor CRISPR RNA (pre-crRNA) sequence were fused with a GAAA or TGAA linker to form a sgRNA sequence (Supplementary Fig. 1). sgRNAs were transcribed under the control of the U6 promoter. We used addgene plasmid (\# 61591) for SaCas 9 expression.

In vitro PAM identification. The recombinant Cas9 protein was expressed in E. coli and purified as described previously ${ }^{3}$. sgRNAs were transcribed using T7 RNA polymerase as described ${ }^{3}$. To make a randomized PAM $\left(\mathrm{N}_{10}\right)$ library, the DNA sequence including the AAVS1-TS1 target site cloned in a plasmid was amplified with a randomized primer. After gel purification, the amplicon library $(1 \mu \mathrm{g})$ was digested with the SpCas9 or CjCas9 protein and in vitro transcribed sgRNA for $30 \mathrm{~min}$ at $37^{\circ} \mathrm{C}$. Digested library was purified by column filtration and subjected to deep sequencing using Miseq (Illumina). Miseq reads that perfectly matched the reference sequence were sorted. The randomized PAM region was extracted and analysed with WebLogo.

PAM characterization using cell-based reporter assays. The AAVS1-TS1 target sites with variable PAM sequences, which were randomized at position X (5'-NNNNXCAC- $3^{\prime}, 5^{\prime}$-NNNNAXAC- $3^{\prime}, 5^{\prime}$-NNNNACXC- $3^{\prime}$ and $5^{\prime}$-NNNN ACAX- $3^{\prime}$ ), were synthesized (Macrogen, Inc.) and cloned in a surrogate reporter plasmid encoding RFP and GFP ${ }^{19}$. To determine optimal PAM sequences, each of the resulting reporter plasmids (100 ng) and plasmids encoding CjCas9 and its sgRNA (225 and $675 \mathrm{ng}$, respectively) were co-transfected into HEK293 cells $\left(1 \times 10^{5}\right)$ using lipofectamine 2000 (Invitrogen). At day 2 post-transfection, the fraction of GFP and RFP double-positive cells was determined by flow cytometry (BD Accuri C6, BD).

Cell culture and mutation analysis. HEK 293 (ATCC, CRL-1573) cells and mouse NIH 3T3 (ATCC, CRL-1658) cells were maintained in DMEM supplemented with 100 units per ml penicillin, $100 \mathrm{mg} \mathrm{ml}^{-1}$ streptomycin, and $10 \%$ fetal bovine serum (FBS). sgRNA plasmid ( $750 \mathrm{ng}$ ) and CjCas9 plasmid ( $250 \mathrm{ng}$ ) were transfected into cells $\left(0.5 \sim 1 \times 10^{5}\right)$ with lipofectamine 2000 (Invitrogen). After $48 \mathrm{~h}$ of transfection, genomic DNA was isolated using a DNeasy Blood \& Tissue kit (Qiagen) and on-target or off-target loci were amplified using specific primers (Supplementary Table 4) for targeted deep sequencing. Deep-sequencing libraries were generated by PCR. TruSeq HT Dual Index primers were used to label each sample. Pooled libraries were subjected to paired-end sequencing (LAS, Inc.). Indel frequencies were calculated as described previously ${ }^{20}$.

Digenome sequencing. Digenome-seq was performed as described previously ${ }^{20,21}$. Genomic DNA was isolated using a DNeasy Tissue kit (Qiagen) according to the manufacturer's instructions. Genomic DNA ( $8 \mu \mathrm{g})$ with CjCas9 or SaCas9 protein $(300 \mathrm{nM})$ and sgRNA $(900 \mathrm{nM})$ in a $400 \mu \mathrm{l}$ reaction volume $\left(100 \mathrm{mM} \mathrm{NaCl}, 50 \mathrm{mM}\right.$ Tris- $\mathrm{HCl}, 10 \mathrm{mM} \mathrm{MgCl}_{2}$, and $\left.100 \mu \mathrm{g} \mathrm{ml}^{-1} \mathrm{BSA}\right)$ and incubated the mixture for $8 \mathrm{~h}$ at $37^{\circ} \mathrm{C}$. Digested genomic DNA was incubated with RNase A $\left(50 \mu \mathrm{gl}^{-1}\right)$ for $30 \mathrm{~min}$ at $37^{\circ} \mathrm{C}$ and purified again with a DNeasy Tissue kit (Qiagen). Digested DNA was fragmented using the Covaris system and ligated with adaptors for library formation. DNA libraries were subjected to WGS using an Illumina HiSeq X Ten Sequencer at Macrogen. We used the Isaac aligner to generate a Bam file using the following parameters: ver. 01.14.03.12; Human genome reference, hg19 from UCSC (original GRCh37 from NCBI, Feb. 2009), Mouse genome reference, mm10 from UCSC; Base quality cutoff, 15; Keep duplicate reads, yes; Variable read length support, yes; Realign gaps, no; and Adaptor clipping, yes (adaptor: $5^{\prime}$-AGATCGGAAGAGC ${ }^{*}-3^{\prime}, 5^{\prime}-{ }^{*}$ GCTCTT CCGATCT $\left.-3^{\prime}\right)^{33}$

AAV vectors encoding CjCas9 and its sgRNA sequences. AAV inverted terminal repeat-based vector plasmids carrying a sgRNA sequence and the CjCas9 gene with a nuclear localization signal and an HA tag at the $\mathrm{C}$ terminus were constructed. sgRNA transcription was driven by the U6 promoter and CjCas9 expression was controlled by the EFS promoter in $\mathrm{C} 2 \mathrm{C} 12$ myoblast cells or by the Spc512 promoter in TA muscles of C57BL6J mice mice. For retinal delivery, an $\mathrm{AAV}$ vector encoding CjCas 9 under the control of the EFS promoter, enhanced green fluorescent protein (eGFP) linked to the C terminus of CjCas9 with the self-cleaving T2A peptide, and a U6 promoter-driven sgRNA specific to the Vegfa or Hifla gene was constructed.

Production and characterization of AAV vectors. To produce AAV vectors, they were pseudotyped in AAVDJ or AAV9 capsids. HEK293T cells were transfected with pAAV-ITR-CjCas9-sgRNA, pAAVED2/9 and helper plasmid. HEK293T cells were cultured in DMEM with 2\% FBS. Recombinant pseudotyped AAV vector stocks were generated using PEI coprecipitation with PEIpro (Polyplus-transfection) and triple-transfection with plasmids at a molar ratio of 1:1:1 in HEK293T cells. After $72 \mathrm{~h}$ of incubation, cells were lysed and particles were purified by iodixanol (Sigma-Aldrich) step-gradient ultracentrifugation. The number of vector genomes was determined by quantitative PCR.

AAV transduction in mouse myoblast cells. Mouse myoblast cells were infected with AAVDJ-CjCas9 at different viral doses (multiplicity of infection (MOI): $1,5,10,50$, and 100 determined by quantitative PCR) and maintained in DMEM with 2\% FBS. At different time points, cells were collected for targeted deep sequencing. An MOI of 1 was estimated with one infectious virus particle in 100 total viral particles determined by quantitative PCR.

Intramuscular injection of AAV. AAV was administered to 8-week-old young adult male C57BL/6J mice anaesthetized with $2-4 \%$ isoflurane. The mice were injected intramuscularly with AAV9-CjCas $9\left(1 \times 10^{11}\right.$ viral genome $)$ in physiological saline $(40 \mu \mathrm{l})$ using an ultra-fine insulin syringe with a $31 \mathrm{G}$ needle (BD). As a negative control, C57BL/6J mice were injected with physiological saline $(40 \mu \mathrm{l})$ only.

Intravitreal injection of AAV. 8-week-old mice were anaesthetized with an intraperitoneal injection of a mixture of tiletamine and zolazepam $(1: 1,2.25 \mathrm{mg}$ per $\mathrm{kg}$ body weight) and xylazine hydrochloride $(0.7 \mathrm{mg}$ per $\mathrm{kg}$ body weight). AAV9-CjCas9 $\left(2 \times 10^{10}\right.$ viral genome in $\left.2 \mu \mathrm{l}\right)$ was intravitreally injected using a Nanofil syringe with a $33 \mathrm{G}$ blunt needle (World Precision Instruments Inc.) under an operating microscope (Leica Microsystems Ltd.).

Immunofluorescent staining and imaging of retinal tissue. For the analysis of opsin-positive area, formalin-fixed paraffin-embedded samples were prepared at day 42 post injection $(n=4)$. Cross-section samples were immunostained with anti-HA antibody (Roche, 3F10, 1:1,000), anti-opsin antibody (Millipore, AB5405, 1:1,000), and Alexa Fluor 488 or 594 antibodies (Thermo Fisher Scientific, 1:500) The opsin-positive area corresponding to RPE cells expressing HA-tagged CjCas9 was measured using Image J software $(1.47 \mathrm{v}, \mathrm{NIH})$ by blinded observers. For the distribution of CjCas9 and eGFP, the eyes were fixed in $4 \%$ paraformaldehyde for $1 \mathrm{~h}$ at room temperature. RPE complexes (RPE/choroid/sclera) were prepared for immunostaining and then incubated with anti-GFP antibody (Abcam, ab6556, 1:100) overnight at $4{ }^{\circ} \mathrm{C}$. After stain with Alexa Fluor 488 antibodies (1:500), the RPE flat-mounts was imaged using a confocal microscope (LSM 710, Carl Zeiss) The scanning parameters were as follows: scaling $(x=0.042 \mu \mathrm{m}$ per pixel, $y=0.042 \mu \mathrm{m}$ per pixel, $z=0.603 \mu \mathrm{m}$ per pixel), dimensions ( $x=1,024, y=1,024$, channels: 2 , 8-bit) with objective C-Apochromat $\times 40$ per 1.20 W Korr M27. ZEN 2 software was used to process the images.

Figure 6 | CjCas9 targeted to Vegfa or Hifla reduces the area of laser-induced CNV in mice. (a) At day 42 post injection of AAV-CjCas9, mice was treated with laser to induce choroidal neovascularization (CNV). One week after laser treatment, the CNV area was analysed. (b) In vivo expression of eGFP coexpressed with CjCas9 in laser-induced CNV. Representative confocal images of eGFP expression in the RPE of laser-induced CNV, 6 weeks after injection of AAV-CjCas9: Hifla. eGFP was stained with anti-GFP antibody (green), CNV was stained with anti-IB4 antibody (red), and nuclei were counterstained with DAPI (blue). Scale bar, $200 \mu \mathrm{m}$. Arrows indicate eGFP-expressing RPE cells. Scale bar, $100 \mu \mathrm{m}$ (enlarged image). (c) Representative laserinduced CNV stained with isolectin B4 in the mouse eye injected with AAV-CjCas9 targeted to Rosa26, Vegfa or Hif1a. Scale bar, $200 \mu$ m. (d) The CNV area. Error bars indicate s.e.m. $(n=17-18)$. One-way ANOVA and Tukey's post hoc tests, ${ }^{\star} P<0.05 ;{ }^{\star \star} P<0.01 ;{ }^{* \star \star} P<0.001$; NS, not significant. (e,f) At day 56 post AAV injection, full-field electroretinogram (ERG) was performed to evaluate cone function in mice. There was no significant decrease of $b$-wave of photopic response (e) and $30 \mathrm{~Hz}$ flicker response $(\mathbf{f})$ in both AAV-CjCas9: Vegfa $(n=8)$ or AAV-CjCas9: Hifla $(n=6)$ treated mice, compared to normal control mice $(n=8)$. Error bars indicate s.e.m. $(n=6-8)$. $(\mathbf{g}, \mathbf{h})$ Opsin-positive areas in the retina at day 42 post injection. (g) Representative images of opsin-positive areas in contact with RPE cells expressing HA-tagged CjCas9 in AAV-CjCas9: Rosa26, Vegfa or Hif1a injected mice compared with the AAV-uninjected negative control mice (no AAV). Opsin (red) and DAPI (blue). Scale bar, $20 \mu \mathrm{m}$. ONL, outer nuclear layer; IS, inner segment of photoreceptor cells; OS, outer segment of photoreceptor cells. See also Supplementary Fig. 6. (h) Relative opsin areas of the AAV-CjCas9-injected mice were normalized to that of the AAV-uninjected negative control mice. Error bars indicate s.e.m. $(n=4)$. One-way ANOVA and Tukey's post hoc tests, ${ }^{\star} P<0.05$. 
Genomic DNA extraction. For DNA extraction from muscle, muscle tissue was homogenized using tungsten carbide beads ( $3 \mathrm{~mm}$; Qiagen) and a TissueLyser II (Qiagen). For extraction from RPE, after imaging of RPE flat-mounts, tissue samples were washed in PBS. RPE cells were mechanically isolated from choroid/ sclera by vortexing for $30 \mathrm{~s}$ in lysis buffer (NucleoSpin Tissue, Macherey-Nagel), as described $^{34}$. Genomic DNA from the remnant choroid/sclera tissues was analysed to confirm complete isolation of RPE cells. Genomic DNA was analysed by targeted deep sequencing.

Mouse Vegfa ELISA. At day 42 post injection, whole RPE complexes were separated from neural retina tissue and frozen for further analysis. Sample tissues were lysed with Cell Lysis Buffer $(120 \mu \mathrm{l})(\mathrm{CST}$ \#9803) and Vegfa protein levels were measured using a mouse VEGF Quantikine ELISA kit (MMV00, R\&D systems) according to the manufacturer's instructions.

ERG analysis. Mice were dark-adapted over $16 \mathrm{~h}$. Mice were anaesthetized with an intraperitoneal injection of a mixture of tiletamine and zolazepam (1:1,2.25 mg per $\mathrm{kg}$ body weight) and xylazine hydrochloride ( $0.7 \mathrm{mg}$ per $\mathrm{kg}$ body weight). Pupils were dilated with an eye drop containing phenylephrine $(0.5 \%)$ and tropicamide $(0.5 \%)$. Contact lens electrodes were placed on both eyes with a drop of methylcellose. Full-field ERGs were recorded as described ${ }^{35}$ by using the universal testing and electrophysiologic system 2000 (UTAS E-2000, LKC Technologies, Gaithersburg, MD). The responses were recorded at a gain of $2 \mathrm{k}$ using a notch filter at $60 \mathrm{~Hz}$, and were bandpass filtered between 0.1 and $1,500 \mathrm{~Hz}$. In the light-adapted state (photopic), with a $30 \mathrm{~cd} / \mathrm{m}^{2}$ background light to desensitize the rods and isolate cones, cone responses were recorded in response to single flashed of $0 \mathrm{~dB}$ for photopic response, and a flicker sequence of $30 \mathrm{~Hz}$, averaging 20 responses. The amplitude of the a-wave was measured from the baseline to the lowest negative-going voltage, whereas peak b-wave amplitudes were measured from the trough of the a-wave to the highest peak of the positive b-wave.

Western blotting. The CjCas9 protein expressed in TA muscles of C57BL/6J mice at 8 months after injection of AAV was detected using western blotting. Samples containing equal amounts of protein $(20 \mu \mathrm{g})$ were analysed; Cas9 and GAPDH were detected with an anti-HA high affinity antibody (Abcam, ab9110, 1:5,000) and an anti-GAPDH antibody (Abcam, ab9485, 1:2,500), respectively. Goat anti-rabbit IgG-HRP antibody (Abcam, ab6721, 1:5,000) was used for signal detection. ImageQuant LAS4000 (GE healthcare) was used for digital imaging. Uncropped scans of blots in main figures are presented in Supplementary Fig. 4.

Statistical analysis. No statistical methods were used to predetermine sample size for in vitro or in vivo experiments. All group results are expressed as mean \pm s.e.m., if not stated otherwise. Comparisons between groups were made using the twotailed Student's $t$-test or one-way analysis of variance (ANOVA) and Tukey's post hoc tests for multiple groups. Statistical significance as compared with untreated controls was denoted with ${ }^{*} P<0.05,{ }^{* *} P<0.01,{ }^{* * *} P<0.001$ in the figures and figure legends. Statistical analysis was performed in Graph Pad PRISM 5

Data availability. The deep-sequencing data from this study have been submitted to the NCBI Sequence Read Archive under accession number SRP095501 and SRP095507. The data that support the findings of this study are available from the corresponding author upon reasonable request.

\section{References}

1. Kim, H. \& Kim, J. S. A guide to genome engineering with programmable nucleases. Nat. Rev. Genet. 15, 321-334 (2014).

2. Jinek, M. et al. A programmable dual-RNA-guided DNA endonuclease in adaptive bacterial immunity. Science 337, 816-821 (2012).

3. Cho, S. W., Kim, S., Kim, J. M. \& Kim, J. S. Targeted genome engineering in human cells with the Cas9 RNA-guided endonuclease. Nat. Biotechnol. 31, 230-232 (2013)

4. Cho, S. W., Lee, J., Carroll, D., Kim, J. S. \& Lee, J. Heritable gene knockout in Caenorhabditis elegans by direct injection of Cas9-sgRNA ribonucleoproteins. Genetics 195, 1177-1180 (2013).

5. Mali, P. et al. RNA-guided human genome engineering via Cas9. Science 339, 823-826 (2013).

6. Cong, L. et al. Multiplex genome engineering using CRISPR/Cas systems. Science 339, 819-823 (2013)

7. Wang, H. et al. One-step generation of mice carrying mutations in multiple genes by CRISPR/Cas-mediated genome engineering. Cell 153, 910-918 (2013).

8. Sung, Y. H. et al. Highly efficient gene knockout in mice and zebrafish with RNA-guided endonucleases. Genome Res. 24, 125-131 (2014).

9. Jinek, M. et al. RNA-programmed genome editing in human cells. eLife 2, e00471 (2013)
10. Wu, Z., Yang, H. \& Colosi, P. Effect of genome size on AAV vector packaging. Mol. Ther. 18, 80-86 (2010).

11. Long, C. et al. Postnatal genome editing partially restores dystrophin expression in a mouse model of muscular dystrophy. Science 351, 400-403 (2016).

12. Swiech, L. et al. In vivo interrogation of gene function in the mammalian brain using CRISPR-Cas9. Nat. Biotechnol. 33, 102-106 (2015).

13. Wright, A. V. et al. Rational design of a split-Cas9 enzyme complex. Proc. Natl Acad. Sci. USA 112, 2984-2989 (2015).

14. Zetsche, B., Volz, S. E. \& Zhang, F. A split-Cas9 architecture for inducible genome editing and transcription modulation. Nat. Biotechnol. 33, 139-142 (2015).

15. Chew, W. L. et al. A multifunctional AAV-CRISPR-Cas9 and its host response Nat. Methods 13, 868-874 (2016).

16. Truong, D. J. et al. Development of an intein-mediated split-Cas9 system for gene therapy. Nucleic Acids Res. 43, 6450-6458 (2015).

17. Ran, F. A. et al. In vivo genome editing using Staphylococcus aureus Cas9. Nature 520, 186-191 (2015).

18. Fonfara, I. et al. Phylogeny of Cas9 determines functional exchangeability of dual-RNA and Cas9 among orthologous type II CRISPR-Cas systems. Nucleic Acids Res. 42, 2577-2590 (2013).

19. Kim, H., Um, E., Cho, S. R., Jung, C. \& Kim, J. S. Surrogate reporters for enrichment of cells with nuclease-induced mutations. Nat. Methods 8, 941-943 (2011).

20. Kim, D. et al. Digenome-seq: genome-wide profiling of CRISPR-Cas9 off-target effects in human cells. Nat. Methods 12, 237-243 (2015).

21. Kim, D. et al. Genome-wide analysis reveals specificities of Cpf1 endonucleases in human cells. Nat. Biotechnol. 34, 888 (2016).

22. Hur, J. K. et al. Targeted mutagenesis in mice by electroporation of Cpfl ribonucleoproteins. Nat. Biotechnol. 34, 807-808 (2016).

23. Kleinstiver, B. P. et al. Genome-wide specificities of CRISPR-Cas Cpf1 nucleases in human cells. Nat. Biotechnol. 34, 869-874 (2016).

24. Bae, S., Park, J. \& Kim, J. S. Cas-OFFinder: a fast and versatile algorithm that searches for potential off-target sites of Cas9 RNA-guided endonucleases. Bioinformatics 30, 1473-1475 (2014)

25. Wu, Z., Asokan, A. \& Samulski, R. J. Adeno-associated virus serotypes: vector toolkit for human gene therapy. Mol. Ther. 14, 316-327 (2006).

26. Kwak, N., Okamoto, N., Wood, J. M. \& Campochiaro, P. A. VEGF is major stimulator in model of choroidal neovascularization. Invest. Ophthalmol. Vis. Sci. 41, 3158-3164 (2000).

27. Andre, H., Tunik, S., Aronsson, M. \& Kvanta, A. Hypoxia-inducible factorlalpha is associated with sprouting angiogenesis in the murine laser-induced choroidal neovascularization model. Invest. Ophthalmol. Vis. Sci. 56, 6591-6604 (2015)

28. Kurihara, T., Westenskow, P. D., Bravo, S., Aguilar, E. \& Friedlander, M. Targeted deletion of Vegfa in adult mice induces vision loss. J. Clin. Invest. 122, 4213-4217 (2012).

29. Esvelt, K. M. et al. Orthogonal Cas9 proteins for RNA-guided gene regulation and editing. Nat. Methods 10, 1116-1121 (2013).

30. Anders, C., Niewoehner, O., Duerst, A. \& Jinek, M. Structural basis of PAM-dependent target DNA recognition by the Cas9 endonuclease. Nature 513, 569-573 (2014).

31. Nishimasu, H. et al. Crystal structure of Staphylococcus aureus Cas9. Cell 162, 1113-1126 (2015).

32. Forsythe, J. A. et al. Activation of vascular endothelial growth factor gene transcription by hypoxia-inducible factor 1. Mol. Cell Biol. 16, 4604-4613 (1996).

33. Raczy, C. et al. Isaac: ultra-fast whole-genome secondary analysis on Illumina sequencing platforms. Bioinformatics 29, 2041-2043 (2013).

34. Wei, H., Xun, Z., Granado, H., Wu, A. \& Handa, J. T. An easy, rapid method to isolate RPE cell protein from the mouse eye. Exp. Eye Res. 145, 450-455 (2016).

35. Gresh, J., Goletz, P. W., Crouch, R. K. \& Rohrer, B. Structure-function analysis of rods and cones in juvenile, adult, and aged $\mathrm{C} 57 \mathrm{bl} / 6$ and Balb/c mice. Vis. Neurosci. 20, 211-220 (2003)

\section{Acknowledgements}

This work was supported by IBS-R021-D1 (to J.-S.K.), the Korea Health technology R\&D Project through the Korea Health Industry Development Institute (Grant Number HI16C0426 to S.K.), the Pioneer Research Program of NRF/MEST (2012-0009544 to Je.H.K.), the Bio \& Medical Technology Development Program of the National Research Foundation and MSIP (NRF-2015M3A9E6028949 to Je.H.K.). We thank Kwang-eun Kim for experimental support.

\section{Author contributions}

J.-S.K. and J.H.K. supervised the research. All the other authors performed the experiments. 


\section{Additional information}

Supplementary Information accompanies this paper at http://www.nature.com/ naturecommunications

Competing financial interests: J.-S.K. is a co-founder and shareholder of ToolGen, Inc. D.W.S., K.J.L., M.H.J. and S.K. are employed by ToolGen, Inc. Je.H.K., S.W.P., D.W.S., E.K., and S.K. have filed patent applications based on this work. The remaining authors declare no competing financial interests.

Reprints and permission information is available online at http://npg.nature.com/ reprintsandpermissions/

How to cite this article: Kim, E. et al. In vivo genome editing with a small Cas9 orthologue derived from Campylobacter jejuni. Nat. Commun. 8, 14500 doi: $10.1038 /$ ncomms14500 (2017).
Publisher's note: Springer Nature remains neutral with regard to jurisdictional claims in published maps and institutional affiliations.

(c) (i) This work is licensed under a Creative Commons Attribution 4.0 International License. The images or other third party material in this article are included in the article's Creative Commons license, unless indicated otherwise in the credit line; if the material is not included under the Creative Commons license, users will need to obtain permission from the license holder to reproduce the material. To view a copy of this license, visit http://creativecommons.org/licenses/by/4.0/

(C) The Author(s) 2017 